\title{
Absence of Normal Photic Integration in the Circadian Visual System: Response to Millisecond Light Flashes
}

\author{
Luis Vidal ${ }^{1}$ and Lawrence P. Morin ${ }^{1,2}$ \\ ${ }^{1}$ Department of Psychiatry and 2 Program in Neuroscience, Stony Brook University, Stony Brook, New York 11794
}

\begin{abstract}
Light is the most prominent synchronizing stimulus for circadian rhythms. The circadian visual system responds in accordance with the energy content of photic stimuli longer than a few seconds. Here, as few as three flashes ( $2 \mathrm{~ms}$ each delivered to hamsters over 5 or $60 \mathrm{~min}$ at circadian time 19) elicited large phase advances. Ten or more flashes were required to induce FOS protein in the suprachiasmatic nucleus ( $\mathrm{SCN}$ ), and such induction occurred throughout the entire SCN, as well as outside the nucleus. High-density flash stimulation $(0.5$ s interflash interval) was ineffective, but response increased as the interval increased up to $4 \mathrm{~s}$. In an irradiance response test, phase shifts appeared to be all-or-none with threshold irradiance between 140 and $1070 \mu \mathrm{W} / \mathrm{cm}^{2}$, implying lack of stimulus energy summation. Nevertheless, an irradiance ineffective when delivered as 10 flashes induced phase shifts when given as 100 flashes, but the response was substantially smaller than elicited by 10 flashes, each with $\sim 1 \log$ unit more irradiance. The results also show reduced sensitivity of flash-induced FOS response in the intergeniculate leaflet compared with the SCN, contrary to studies using longer light stimuli. Masking was robust and prolonged in response to 10 flashes. The data demonstrate that the circadian visual system responds markedly to brief, intense light stimuli without normal photic integration. This may involve a second input pathway different from that mediating the effects of longer, dimmer photic stimuli.
\end{abstract}

Key words: suprachiasmatic; intergeniculate; pregeniculate; retina; photoreceptor; retinohypothalamic tract

\section{Introduction}

Mammalian behavioral and physiological circadian rhythms are generated by a clock in the suprachiasmatic nucleus (SCN) of the hypothalamus (Moore and Eichler, 1972; Stephan and Zucker, 1972; Rusak, 1977). Light is the primary entraining stimulus of circadian rhythms, serving to synchronize the SCN clock with the daily environmental photoperiod. Ordinarily, such entrainment is mediated by the combined action of the classical retinal photoreceptors, rods and cones, and photoreceptive retinal ganglion cells (pRGCs) containing the novel photopigment melanopsin (Provencio et al., 2000; Berson et al., 2002; Hattar et al., 2002; Panda et al., 2002; Ruby et al., 2002). The retinohypothalamic tract (RHT), a direct retinal projection to the SCN (Hendrickson et al., 1972; Moore and Lenn, 1972; Meijer et al., 1986; Johnson et al., 1988a,b; Muscat et al., 2003), provides luminance information necessary for entrainment (Meijer et al., 1986; Johnson et al., 1988b).

One unusual property of the circadian visual system is its ability to integrate photic input over a relatively long period of time with rhythm phase response proportional to the energy of the stimulus (Takahashi et al., 1984; Nelson and Takahashi, 1991;

Received Dec. 19, 2006; revised Jan. 29, 2007; accepted Feb. 16, 2007

This work was supported by National Institutes of Health Grants NS22168 and MH64471 (L.P.M.). We thank Jane Blanchard for her excellent technical assistance.

Correspondence should be addressed to Dr. Lawrence P. Morin, Department of Psychiatry, Health Science Center, Stony Brook University, Stony Brook, NY 11794-8101. E-mail: lawrence.morin@stonybrook.edu.

L. Vidal's present address: Department of Physiology and Pharmacology, Harris Hall H203, City College of New York, 138th Street and Convent Avenue, New York, NY 10031.

D01:10.1523/JNEUROSCI.5496-06.2007

Copyright $\odot 2007$ Society for Neuroscience $\quad$ 0270-6474/07/273375-08\$15.00/0
Dkhissi-Benyahya et al., 2000; Muscat and Morin, 2005). The stimulus intensity threshold for eliciting phase shifts is fairly high, and minimum effective stimulus duration is relatively long. Photic stimulation methods for eliciting rhythm phase shifts typically use a continuous light stimulus with a duration ranging from minutes to hours (a light "pulse"). Data obtained with these methods support the conclusion that very brief stimuli cannot provide the necessary energy to elicit circadian rhythm phase response (Takahashi et al., 1984; Nelson and Takahashi, 1991). Thus, it was surprising when van den Pol et al. (1998) demonstrated that mice could respond with robust phase shifts to a series of millisecond duration light stimuli ("flashes"). Rats also respond to millisecond (or briefer) light flashes with both rhythm phase shifts and FOS protein induced in the SCN (Arvanitogiannis and Amir, 1999).

The present experiments were designed to study the photic integration response of the circadian visual system to millisecond light stimuli. The absence of normal photic integration in the studies is discussed with respect to the known properties of the three classes of photoreceptor cells. Additionally, light flashinduced FOS in the SCN has been evaluated to determine whether the density or pattern within the SCN differed from that induced by saturating 5 min light pulses.

\section{Materials and Methods}

Animals. Adult, outbred male golden hamsters weighing 90-100 g (Charles River Laboratories, Wilmington, MA) were housed in individual plastic cages and maintained under a 14/10 h light/dark photoperiod (LD 14:10) with access to food and water ad libitum. All experimental 
procedures were approved by the Institutional Animal Care and Use Committee of Stony Brook University.

Photic stimulation. For all experiments, each animal was placed in a home cage containing a running wheel and allowed to stably entrain to an LD 14:10 photoperiod $\sim 3$ weeks before release into constant darkness (DD). One week later, the animals were removed from their home cages and placed in a photic stimulation chamber. Light stimulation was centered on circadian time 19. At approximately $5 \mathrm{~min}$ after stimulation, the animals were returned to their home cages for $14 \mathrm{~d}$ in DD (except for those with brains to be evaluated for FOS protein immunoreactivity). Animals subjected to the stimulation sequence more than once were re-entrained to the original $\mathrm{LD}$ 14:10 for at least $3 \mathrm{wks}$ after the $14 \mathrm{~d}$ in DD.

The procedure for exposing animals to a continuous $5 \mathrm{~min}$ light pulse of saturating intensity $\left(57 \mu \mathrm{W} / \mathrm{cm}^{2}\right)$ has been described previously (Muscat and Morin, 2005). Briefly, on the day of stimulation, animals (up to eight simultaneously) were transferred to individual, clear acrylic cylinders and placed in the stimulus chamber. Illumination was provided by an Eastman Kodak (Rochester, NY) carousel projector under computer control. The projector was fitted with infrared/ultraviolet filters to cut wavelengths below $380 \mathrm{~nm}$ and above $680 \mathrm{~nm}$. Stimulus irradiance was controlled via neutral density filters selected by computer, and duration was computer controlled.

For flash stimulation, animals were placed in a light-tight stimulus chamber measuring $\sim 0.9 \times 0.9 \times 1.25 \mathrm{~m}$ high. On the day of stimulation, animals (up to 14 simultaneously) were transferred to individual, clear acrylic cylinders placed on the chamber bottom. Timing and number of flashes were computer controlled. Flashes of white light were generated by a Dyna-Lite (Union, NJ) Flash Head (model 2040) placed into an opening through the top of the stimulus chamber. The Dyna-Lite Flash Head was powered by a Dyna-Lite M1000er power supply; the same combination as used by van den Pol et al. (1998). The pulse width of each flash was $\sim 2 \mathrm{~ms}$, as indicated by the specifications of the manufacturer and measured $\sim 4 \times 10^{3} \mu \mathrm{W} / \mathrm{cm}^{2}\left(0.08 \mathrm{~J} / \mathrm{m}^{2}\right)$. Unless otherwise stated, animals were exposed directly to the flashes from the Dyna-Lite Flash Head without intervening filters or leaf diaphragm (as did van den Pol et al., 1998). When variation in flash irradiance was necessary, neutral density filters and a leaf diaphragm were placed in the light path. Irradiance levels for all experiments were verified with a Gigahertz-Optik (Newburyport, MA) P-9710 photometer that has the capability of measuring millisecond light stimuli. Infrared emission during each flash was pronounced, but the extent to which infrared or ultraviolet energy influenced the photometer measurements is unknown. The infrared light itself is unlikely to have had an effect on phase response (Takahashi et al., 1984; Provencio and Foster, 1995; Hattar et al., 2003), although it is possible that ultraviolet emissions contributed to the observed phase shifts (Jacobs et al., 1991; Amir and Robinson, 1995). In addition, no attempt was made to ensure that each animal was exposed to the photic stimuli in an identical manner. Thus, if an animal was facing away from the light source or had its eyes closed, the actual stimulus would differ from the measured value and contribute to the experimental variability.

Running rhythms and phase shift measurement. Hamster wheel running was monitored and collected by a computer in 1 min bins. This information was further reduced to $5 \mathrm{~min}$ bins and plotted in typical raster format. On completion of the poststimulus $14 \mathrm{~d}$ period in DD, activity onsets and phase shifts were determined by visual estimation, as described by Muscat and Morin (2005), with phase shift magnitude determined as the time difference, calculated on the day of stimulation, between activity onset before the light stimulus and extrapolated phase of activity onset during at least $5 \mathrm{~d}$ of stable data after the stimulus. Phase shifts were measured to the nearest $0.01 \mathrm{~h}$.

FOS immunohistochemistry. Each anesthetized animal was perfused transcardially with physiological saline, $\mathrm{pH} 6.6$, followed by $4 \%$ paraformaldehyde in $0.1 \mathrm{M}$ phosphate buffer, $\mathrm{pH} 7.4$, with $0.01 \mathrm{M}$ sodium periodate and $0.075 \mathrm{~g}$ of lysine added. Each brain was removed and postfixed overnight in the same fixative at $4^{\circ} \mathrm{C}$. The brains were cryoprotected in $30 \%$ sucrose in phosphate buffer, frozen, and serially sectioned $(30 \mu \mathrm{m})$ in the coronal plane on a freezing stage microtome. Four series of sections were collected in $0.01 \mathrm{M}$ PBS, pH 7.2, with $0.05 \%$ sodium azide. All immunohistochemical reactions were performed using free-floating sections. Tissue sections were incubated in primary antibody (rabbit antiFOS, 1:20,000; Oncogene Research Products, San Diego, CA) for 36-72 h at $4^{\circ} \mathrm{C}$. Immunoreactivity was visualized using the avidin-biotin complex technique (Elite kit; Vector Laboratories, Burlingame, CA) with $\mathrm{DAB} /$ nickel as the chromogen. Processing of the tissue from the various treatment groups was balanced to minimize processing errors.

Analysis of FOS-immunoreactive (IR) nuclei was performed by a Zeiss (Oberkochen, Germany) microscope system. This system consisted of a computer with Axio Vision 4.3 image software coupled to an Axioplan 2 microscope via a high-resolution AxioCam HR camera. Counts of FOS-IR nuclei in cells of the SCN and intergeniculate leaflet (IGL) were performed as described previously (Muscat and Morin, 2005). Briefly, images were digitally photographed in grayscale with a fixed illumination level. Previously created templates were used to outline the SCN and IGL at the different rostrocaudal levels. For each digital image, the most appropriate template was positioned and superimposed using Corel (Ottawa, Ontario, Canada) Photo-Paint 12. The area within the SCN and IGL boundaries was cut and transferred to NIH Image J (http://rsb.info.nih.gov/ij/) to automatically count labeled cells in the selected region of interest as defined by the template. For each brain, the estimated counts from each of the five sections per SCN or eight sections per IGL were summed to determine a final cell count of each nucleus in that brain.

One analysis used a $4 \times 7$ array of squares, each square being a $90 \times 90$ $\mu \mathrm{m}$ template, placed to encompass most of the SCN plus adjacent hypothalamus. The same tissue used to count FOS-IR nuclei within the SCN was analyzed on a per square basis, using the methods described above, at each level of the SCN with the medial border of the array $45 \mu \mathrm{m}$ from the midline. No effort was made to accommodate between-brain variation in tissue tears, distortion, or size of the third ventricle. Because of such issues and the variability between brains, the value of this analysis lies in the broader perspective it provides rather than in narrower, square-bysquare results. The array analysis involved a large number of statistical tests. Therefore, the level of significance by ANOVA was set at $p<0.025$.

Statistical analysis. Parametric or nonparametric ANOVA and appropriate post hoc tests were used to assess effects of treatments, depending on initial analysis of distribution normality. The software package SigmaStat (SPSS, Chicago, IL) was used for the statistical analysis.

Experiment 1: flash density, stimulus delivery over 5 or 60 min intervals. Animals were exposed to a number of light flashes that varied from 0 to 100 per group. The flashes were equally distributed over a 5 or $60 \mathrm{~min}$ interval. An additional group was exposed to the saturating $5 \mathrm{~min}$ continuous light pulse and served as a standard control.

Experiment 2: flash density, stimulus delivery over short intervals. Experiment 1 determined that 10 flashes delivered over a 5 min interval induced a near-maximal phase response in all animals. This stimulus was designated as the "standard flash stimulus" and was used in the present experiment. Animals were exposed to 10 flashes equally distributed over one of the following intervals: 5, 10, 20, 40, 80, 160, or $300 \mathrm{~s}$.

Experiment 3: phase response to light flash irradiance. On the day of stimulation, animals were randomly assigned to a specific intensity group and exposed to the standard flash stimulus. Flash irradiance varied from $0.42 \mu \mathrm{W} / \mathrm{cm}^{2}$ to a device maximum of $4.35 \times 10^{3} \mu \mathrm{W} / \mathrm{cm}^{2}$, according to group, in steps of $\sim 1 \log$ unit. The irradiance levels were achieved with a combination of change in diaphragm aperture and neutral density filters.

Experiment 4: light integration and stimulus reciprocity. Experiment 1 revealed that at least 10 flashes over 5 min were necessary to elicit a consistent phase shift in $100 \%$ of the animals. Furthermore, results from experiment 2 showed an irradiance difference between effective and ineffective flashes of $1 \log$ unit or less. In the present experiment, a flash stimulus series with an irradiance below threshold was used to test whether an increase in the number of flashes would compensate for the lower irradiance of the stimulus, thereby allowing the two different stimulus series to elicit equivalent phase shifts. These results were compared with the effects of a flash series identified in experiment 1 as effectively eliciting phase shifts. On the day of stimulation, hamsters were randomly assigned to a group that received (1) 10 flashes $/ 5 \mathrm{~min}\left(89 \mu \mathrm{W} / \mathrm{cm}^{2}\right.$ per flash), (2) 100 flashes $/ 5 \mathrm{~min}\left(89 \mu \mathrm{W} / \mathrm{cm}^{2}\right.$ per flash), or (3) 10 flashes $/ 5$ 


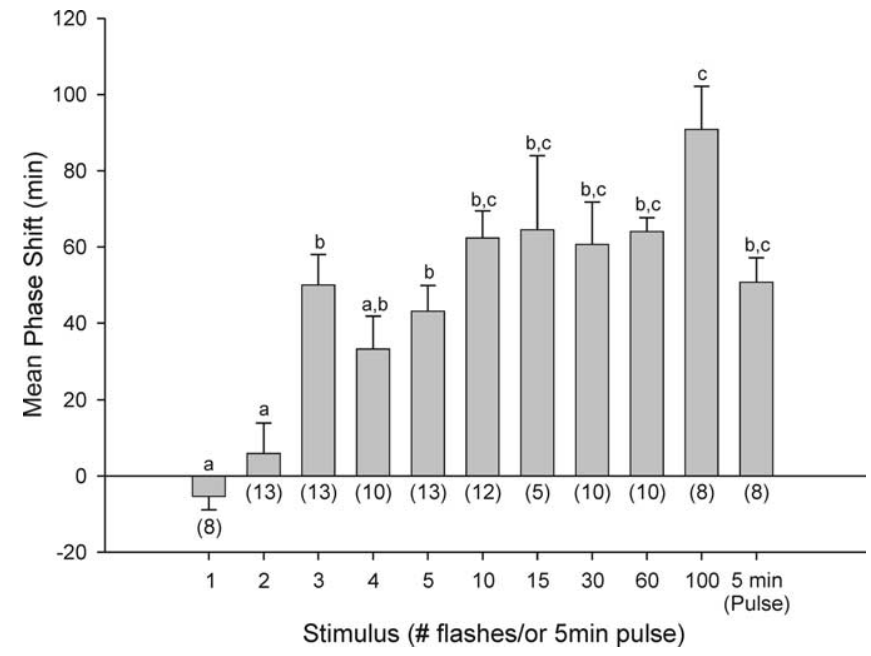

Figure 1. Mean \pm SEM phase response to various numbers of flashes delivered across a 5 min interval. Groups with different letters are significantly different $(p<0.05)$. $n$ per group is indicated in parentheses.

$\min \left(830 \mu \mathrm{W} / \mathrm{cm}^{2}\right.$ per flash). Thus, both groups 2 and 3 received $\sim 10$ times the total energy received by group 1 .

Experiment 5: flash-induced FOS protein in the SCN and IGL. The number of flashes delivered per $5 \mathrm{~min}$ interval varied from 0 to 100 , according to group. Additional animals were exposed to a 5 min continuous pulse of saturating light $\left(\sim 57 \mu \mathrm{W} / \mathrm{cm}^{2}\right)$. Approximately $90 \mathrm{~min}$ after photic stimulation, each animal was deeply anesthetized with sodium pentobarbital (100 mg/kg body weight; Nembutal sodium solution; Abbott Laboratories, North Chicago, IL), perfused as described above, and its brain was prepared for histology.

Experiment 6: evaluation of flash-induced masking. Animals were caged with running wheels in LD 14:10 until entrainment was stable. At this time, animals in their cages were moved to the floor of the stimulus apparatus at Zeitgeber time 2 (ZT2) to ZT4, in which they remained until at least ZT16, while wheel running was monitored. At ZT14, the animals were exposed to the standard flash stimulus. The wheel revolutions during a $65 \mathrm{~min}$ interval, starting $5 \mathrm{~min}$ before the first flash, were assessed for each animal. Data for control animals were assessed during the corresponding interval during which the flash head was not activated or was covered with an opaque material and activated (the two procedures yielded equivalent control results). One control and one experimental animal failed to run during the $5 \mathrm{~min}$ baseline, and their data were not included in the analysis.

\section{Results}

\section{Experiment 1: flash density, stimulus delivery over 5 or 60} min intervals

There was a significant effect of flash density during a 5 min interval on phase shift magnitude $(p<0.001)$. Exposure to one or two flashes had little or no effect on the phase response (Fig. 1), and the responses did not differ significantly (Tukey's post hoc test, $p>0.05$ ). Robust phase shifts were observed in response to as few as three flashes ( $p<0.05$ vs one or two flashes), although shifts were not observed in all individuals unless 10 or more flashes were presented. The largest phase advance ( $\sim 90 \mathrm{~min})$ was observed after a series of 100 flashes. This stimulus elicited a phase response that was significantly greater than that elicited by five or fewer flashes per $5 \mathrm{~min}(p<0.05)$ but not different from responses to the other flash stimuli or to the $5 \mathrm{~min}$ light pulse $(p>0.05)$.

There was also a significant effect of flash density during a 60 min interval on phase shift magnitude $(p<0.001)$. Two flashes elicited a small phase response (Fig. 2), with more robust re-

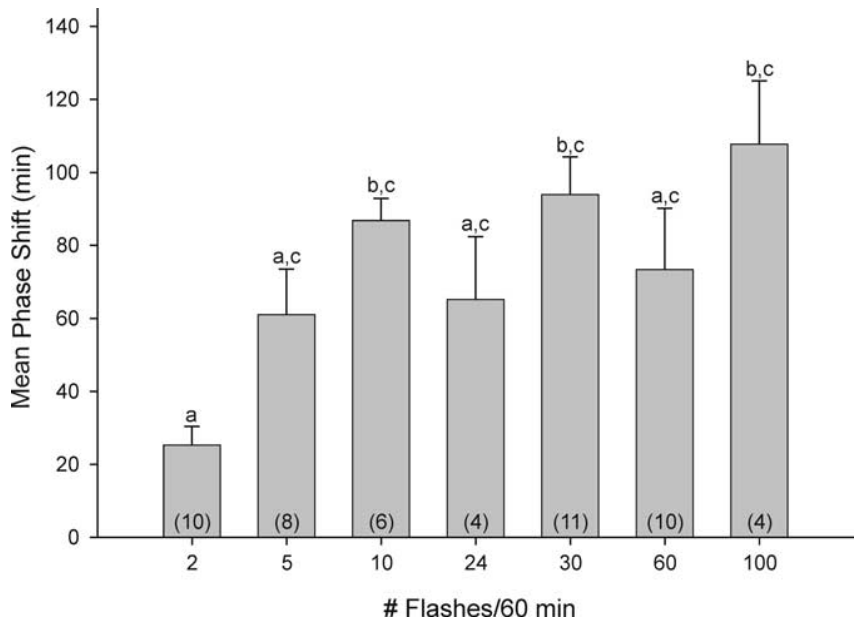

Figure 2. Mean \pm SEM phase response to various numbers of flashes delivered across a 60 min interval. Groups with different letters are significantly different $(p<0.05)$. $n$ per group is indicated in parentheses.

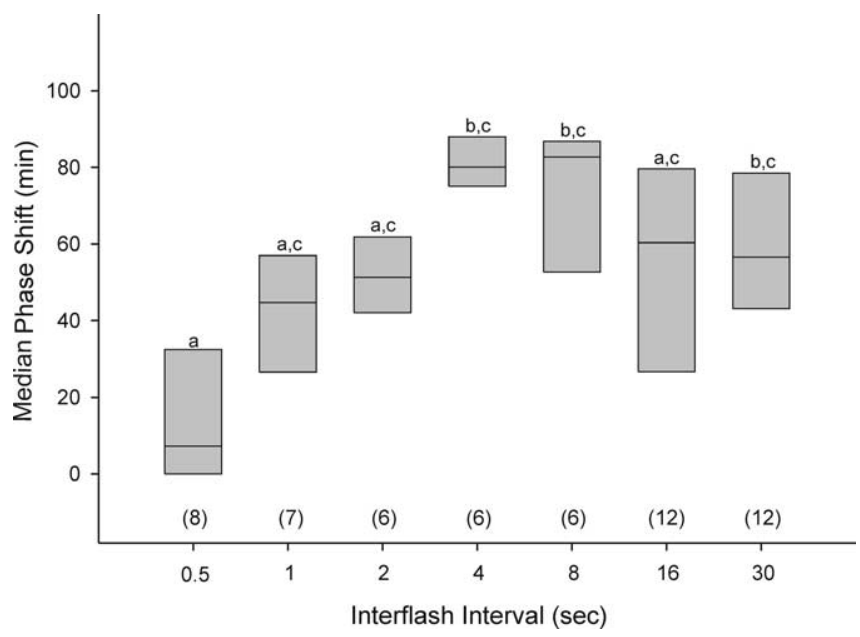

Figure 3. Median phase response to 10 flashes delivered with various interflash intervals. The horizontal line in each gray box indicates the median shift; upper and lower ends of the box represent the interquartile range and the 75 th and 25 th percentiles, respectively. Groups with different letters are significantly different $(p<0.05)$. $n$ per group is indicated in parentheses.

sponses observed after five or more flashes per $60 \mathrm{~min}$. Pairwise analysis detected significant differences between groups that received 10, 30, and 100 versus 2 flashes (Tukey's test, $p<0.05$ ). Although there was a trend toward increased phase response with an increase in flash number, there was no significant difference in phase response between any two groups receiving five or more flashes.

\section{Experiment 2: flash density, stimulus delivery over short intervals}

Phase shifts in response to the various series of flashes were not normally distributed. An ANOVA on ranks revealed a significant effect of interflash interval on phase response (Fig. 3) (KruskallWallis ANOVA, $p=0.004$ ). Animals receiving a flash every $0.5 \mathrm{~s}$ showed little or no phase response, but response increased progressively to a maximum with a $4-8 \mathrm{~s}$ interflash interval. Multiple pairwise comparisons revealed that 10 flashes presented every 4 or $8 \mathrm{~s}$ produced a phase response that was significantly greater than that to flashes every $0.5 \mathrm{~s}$ (Dunn's post hoc test, $p<0.05$ ). 


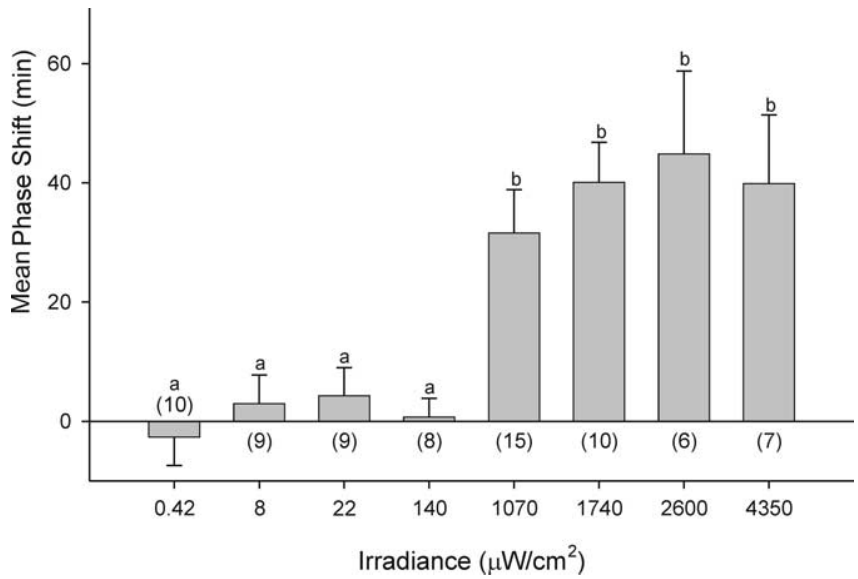

Figure 4. Mean \pm SEM phase response to a series of flashes set at various irradiances. Groups with different letters are significantly different $(p<0.05)$. $n$ per group is indicated in parentheses.

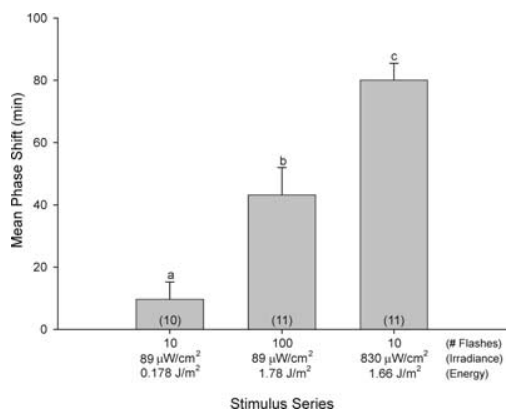

Figure 5. Mean \pm SEM phase response to three different series of flash stimuli. The stimulus eliciting the response shown by the middle bar is $\sim 1 \log$ unit greater than that eliciting the response shown in the left bar. The stimulus eliciting the response shown by the right bar is slightly less than that corresponding to the middle bar, although the response is much greater. Groups with different letters are significantly different $(p<0.05)$. $n$ per group is indicated in parentheses.

\section{Experiment 3: phase response to light flash irradiance}

Phase response to 10 flashes $/ 5$ min generated an irradiance response curve of the all-or-none variety. Phase shift magnitude varied with stimulus irradiance (Kruskall-Wallis ANOVA, $p<$ $0.001)$, but if irradiance was $140 \mu \mathrm{W} / \mathrm{cm}^{2}$ or less, phase shifts were small to nonexistent (Fig. 4). In contrast, the higher irradiances tested ( $1070 \mu \mathrm{W} / \mathrm{cm}^{2}$ or greater) elicited reliable, equivalent, and statistically significant (Dunn's post hoc test, $p<0.05$ compared with the lower irradiances) phase shifts, although the average shift was somewhat smaller than was typical in other experiments.

\section{Experiment 4: light energy integration and stimulus reciprocity}

A subthreshold stimulus of 10 flashes $/ 5 \mathrm{~min}$, with irradiance fixed at $89 \mu \mathrm{W} / \mathrm{cm}^{2}$ per flash, produced a small phase shift (Fig. 5). When the number of flashes was raised to 100 flashes $/ 5 \mathrm{~min}$ with the same irradiance, a significant phase advance occurred. An increase of the flash irradiance by $\sim 1 \log$ unit for a series of 10 flashes $/ 5$ min elicited an even larger phase shift than produced by 100 flashes at the lower irradiance.

Experiment 5: flash-induced FOS protein in the SCN and IGL FOS-IR nuclei in the SCN varied according to the number of flashes per 5 min (Figs. 6, 7) (Kruskall-Wallis ANOVA, $p<$
0.001). Exposure to one or two flashes induced little or no FOS compared with no-light controls (Fig. 7). All other stimuli caused significant FOS induction (Dunn's test, $p<0.05$ ), with 60 flash$\mathrm{es} / 5 \mathrm{~min}$ the most effective stimulus. A separate ANOVA plus post hoc tests included the effects of a 5 min continuous light pulse on FOS protein induction. The 5 min pulse induced FOS expression both quantitatively and qualitatively similar to that observed after 60 flashes $/ 5 \mathrm{~min}$ and greater than the response by the no-light or one or two flash groups ( $p<0.05$ for each comparison). FOS counts after a 5 min light pulse were not significantly different from those observed after 10 or more flashes.

Anatomical analysis revealed that SCN sections from animals not exposed to light or to one or two flashes had only a few FOS-IR nuclei, and most of these were in the caudal SCN. The distribution of the FOS-IR nuclei was generally similar for animals that received a 5 min light pulse or 10 or more flashes with the exception that density of FOS-IR nuclei tended to be greater after either a 5 min light pulse or 60 flashes. A 5 min light pulse or 10 or more flashes elevated counts of FOS-IR nuclei everywhere in the SCN, although the amount of FOS induction was not uniform. There are three fairly obvious areas with greater or lesser FOS-IR density. At the midcaudal SCN (Fig. 6, level D), there is a ventral region of dense FOS-immunoreactivity generally correspondent to the area containing a collection of calbindin-IR neurons (Hamada et al., 2001; Lee et al., 2003), although it is clear that the region of denser FOS extends well rostral to most of the calbindin neurons (Fig. 6, levels B, C). Dorsal, medial, and dorsomedial to this region is an area in which FOS is much less obvious (Fig. 6, levels B-D). After 10 flashes $/ 5 \mathrm{~min}$, few cells in this region express FOS. After 60 flashes or a 5 min pulse, FOS is induced within the area, but the expression per cell nucleus is much less, as judged from the darkness of the reaction product. The third region is in the dorsal extension of the SCN, in which the nucleus abuts the subparaventricular hypothalamus. Here, FOS induction is noteworthy as being greater than in the area just ventral to it but not as dense as in the caudocentral part of the nucleus.

Although anatomical detail is lost, the array analysis reveals two major effects of light on the SCN and nearby hypothalamus. One is a significant effect of flash number $(p<0.025)$ on FOS counts in most squares (Fig. $8 A-E$ ). The second is an indication that 60 flashes $/ 5$ min induces an average increase in every square of the array after the average counts of the corresponding no-light control square has been subtracted and that the induction is much greater in some areas than in others. Similar analyses were performed on the SCN region of each animal in each of the flash stimulus groups with generally similar results for each group receiving 10 or more flashes.

Light flashes induced FOS protein expression in the IGL (Fig. 9) (Kruskal-Wallis ANOVA, $p<0.001$ ). FOS immunoreactivity was not induced in IGL cells in animals that received 10 or fewer flashes. FOS induction occurred to some extent in response to 20 or 40 flashes $/ 5 \mathrm{~min}$, but the effect of the flashes was not significantly different from the no-light response unless the animals experienced 60 or 100 flashes or a 5 min light pulse (Dunn's test, $p<0.05)$. Maximal FOS-IR nuclei were counted in animals that received 60 flashes $/ 5 \mathrm{~min}$, but this group did not significantly differ from those receiving 20,40, or 100 flashes or from counts in animals that received a 5 min light pulse.

Anatomical analysis revealed that the sparse FOS-IR nuclei present in the IGL of animals receiving no light, 1, 2, or 10 flashes were scattered throughout the nucleus (Fig. 10). In the groups receiving 20 or more flashes or a $5 \mathrm{~min}$ light pulse, sparse to 


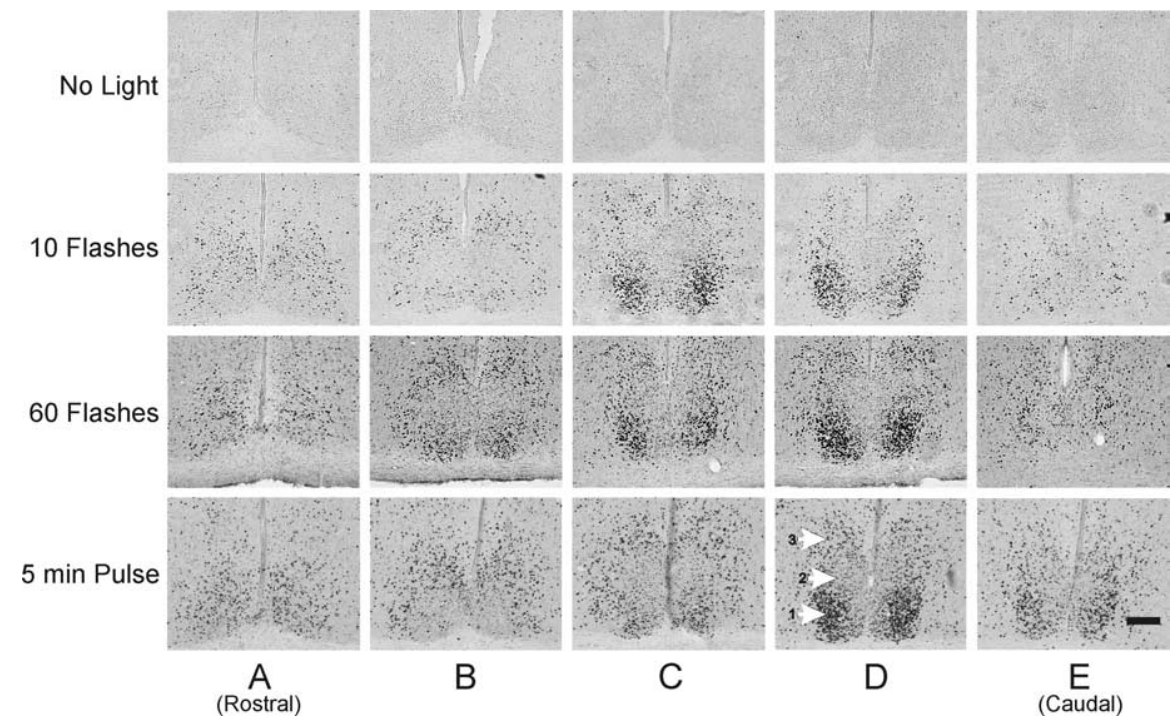

Figure 6. Representative histological cross sections through five SCN levels illustrating of FOS-IR nuclei distribution after flash or pulse stimuli. White arrows identify the three regions of greater (1 and 3) or lesser (2) FOS induction (see Results). Scale bar, $150 \mu \mathrm{m}$.

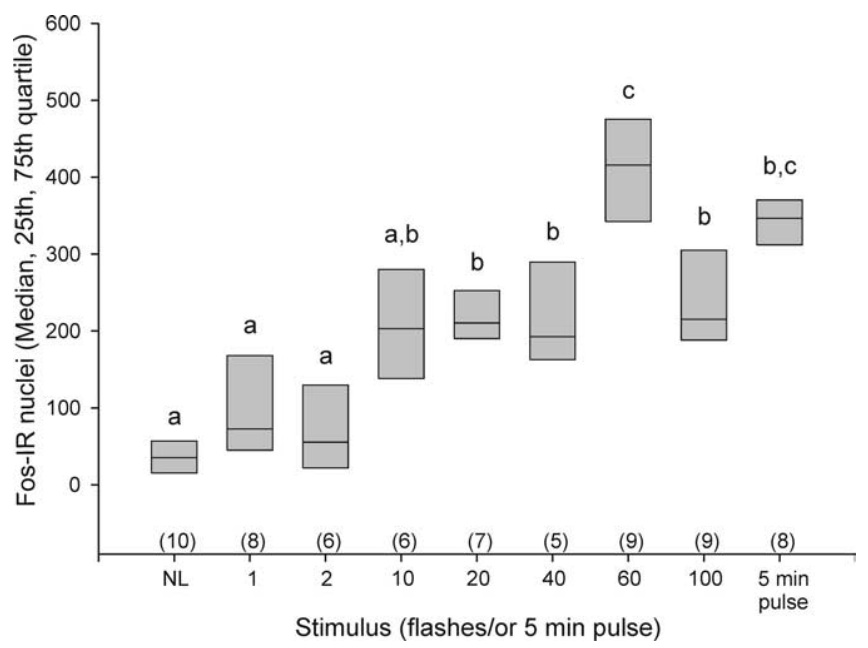

Figure 7. Median number of FOS-IR nuclei in the SCN after stimulation by various numbers of flashes or a 5 min light pulse. Horizontal line in each gray box indicates the median; upper and lower ends of the box represent the interquartile range and the 75th and 25th percentiles, respectively. Groups with different letters are significantly different $(p<0.05)$. $n$ per group is indicated in parentheses.

modest numbers of FOS-IR nuclei were observed throughout the rostral half of the IGL, but the majority were found in the caudal half of the IGL, with most in the dorsolateral and ventromedial portions of the nucleus.

\section{Experiment 6: flash-induced masking}

Flashes were associated with a dramatic decrease in wheel running (Fig. 11). The majority of animals failed to run at all for 35-40 min after the stimulus, although the average revolutions per $5 \mathrm{~min}$ interval gradually increased during this time. By minute 60,8 of 11 animals had resumed running.

\section{Discussion}

Brief light flashes elicit robust phase shifts in hamsters, as has been reported for mice and rats (van den Pol et al., 1998; Arvanitogiannis and Amir, 1999). The present studies expand on previ- ous work and demonstrate that (1) only a few such flashes are necessary, (2) flashes are effective even if distributed over long time intervals, (3) there is a minimum interflash interval of several seconds, and (4) phase response is maximal above the threshold irradiance for an effective flash stimulus. In addition, flashes induce robust masking that endures substantially beyond the stimulus interval and FOS protein in the SCN that is patterned similar to what is seen after a more prolonged light pulse. FOS induction in SCN neurons appears to be more sensitive to flash stimulation than it is in IGL neurons. In some respects, hamsters are able to integrate photic input such that larger phase shifts occur in response to greater flash energy, but the results are substantially different from previous characterizations of integration in which longer duration light pulse stimuli were used.

\section{Integration of photic input}

In neither mouse (van den Pol et al., 1998) nor hamster is a single, high-energy flash sufficient to induce phase shifts, but both species respond robustly to 60 flashes. Thus, some form of integration mechanism is evaluating the adequacy of the stimulus, with one flash being below threshold and three or more being an adequate stimulus for maximal phase response by most individuals. The nature of the mechanism appears to be substantially different from the "photon counting" mechanism inferred from data obtained with the use of much longer and dimmer light stimuli (Nelson and Takahashi, 1991; Dkhissi-Benyahya et al., 2000). The photon counting mechanism they described exhibits reciprocity between stimulus duration and irradiance, with effective stimuli ranging from a few seconds to $>1 \mathrm{~h}$ duration. In the present studies, with one exception, phase shifts only occurred in response to very high flash irradiances, and the irradiance response curve exhibited a step function. Experiment 4 most closely approximated a duration/irradiance reciprocity study, showing that a series of flashes below threshold irradiance becomes effective if the number of flashes is increased 10 times. However, the magnitude of the response to more flashes is not as great as that after a much higher irradiance $(\sim 1 \log$ unit) stimulus consisting of the smaller number of flashes. This result suggests that a limited form of reciprocity and integration of light information is occurring over a fairly small range of flash irradiances or number of flashes.

The threshold for inducing phase shifts lies between 0.028 and $0.214 \mathrm{~J} / \mathrm{m}^{2}$ (10 flashes, $2 \mathrm{~ms}$ each at 140 and $1070 \mu \mathrm{W} / \mathrm{cm}^{2}$, respectively), as determined from experiment 3 and is $\sim 1 \log$ unit less than an estimate obtained in experiment 1 (two vs three flashes; 1.66 vs $2.49 \mathrm{~J} / \mathrm{m}^{2)}$. The difference may be related to how the irradiance was modulated in experiment 3 or to different mechanisms regulating response to flash number and flash irradiance. Regardless, the threshold light flash power that elicits a robust, nearly maximal, phase response would be unlikely to cause more than a minimal phase shift or FOS induction in the $\mathrm{SCN}$, if the same power had been delivered as a light pulse (Muscat and Morin, 2005). 


\section{Photoreceptors}

The physiological properties of classic photoreceptors (i.e., rods and cones) and the melanopsin-containing pRGCs differ substantially. Rods and cones respond rapidly to brief light flashes (Baylor, 1987), whereas pRGCs exhibit a much longer latency to peak response, have sustained depolarization and exhibit little adaptation, ideal for integrating photic input over time (Berson et al., 2002). Rods have been established as contributors to circadian rhythm phase control (Mrosovsky, 2003), but the role of cones is unclear (Hattar et al., 2003). Of particular interest here is the marked reduction in phase response that occurs when the interflash interval is short. This may be at least partially explained by loss of sensitivity resulting from rod activation by each flash and the rate of dark adaptation (recovery of sensitivity) after the flash. A single flash of the type used in the present studies can greatly reduce rod retinal response for a prolonged interval after the flash (Hetling and Pepperberg, 1999). Recovery of rod photoresponse is $\sim 50 \%$ after a flash administered $5 \mathrm{~s}$ after an initial flash (Fulton and Hansen, 2003). The rate is significantly faster than adaptation by pRGCs in which the time course is in minutes rather than seconds (Wong et al., 2005). Alternatively, the circadian visual system may respond to a rapid series of flashes as if it is a single pulse. Short-duration (e.g., $3 \mathrm{~s}$ ) light pulses induce only small shifts (Nelson and Takahashi, 1991) approximately equivalent to what has been observed in response to 10 flashes delivered over $5 \mathrm{~s}$. Whether or not brief, bright light flashes are mediated by rods and/or cones appears to be further complicated by the likelihood that information from the classic photoreceptors is transmitted first to pRGCs before being passed to the circadian rhythm system (Dacey et al., 2005; Ecker et al., 2005). Mice lacking melanopsin have a generally normal scotopic electroretinogram response to light flashes, although details may differ under some lighting conditions (Fu et al., 2005).

\section{Light-induced FOS protein expression}

The threshold stimulus for significant FOS-IR induction in nuclei of SCN neurons was $\sim 10$ standard flashes $\left(8.3 \mathrm{~J} / \mathrm{m}^{2}\right)$. Increases in the number of flashes from 10 to 100 failed to induce a proportional increase in FOS immunoreactivity. This agrees well with the behavioral data indicating that the response is maximal after just 10 flashes. For unknown reasons, the FOS response in both SCN and IGL to 60 flashes was approximately twice that produced by the other effective flash series and equivalent to that observed after a $5 \mathrm{~min}$ saturating light pulse. It is possible that the 60 flash/5 min series directly activates pRGCs and the increased FOS induction represents additive effects of activated pRGCs and the classical photoreceptors.

FOS immunoreactivity was induced by light in all parts of the SCN, consistent with the fact that the entire nucleus receives retinal innervation (Johnson et al., 1988a; Muscat et al., 2003). The region of densest FOS expression also receives very dense RHT terminals, especially from the contralateral eye. The ventral

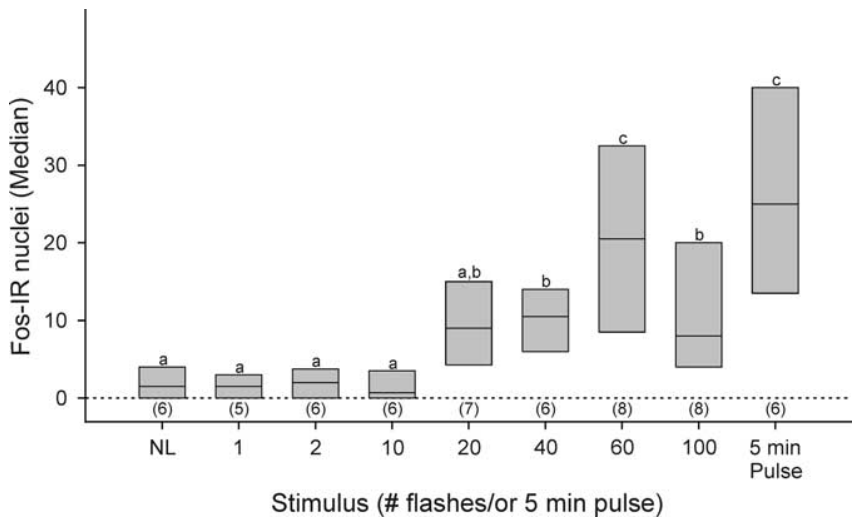

Figure 9. Median FOS-IR nuclei in the IGL after stimulation by various numbers of flashes or a 5 min light pulse. The horizontal line in each gray box indicates the median count; upper and lower ends of the box represent the interquartile range and the 75 th and 25 th percentiles, respectively. Groups with different letters are significantly different $(p<0.05) . n$ per group is indicated in parentheses. Groups with different letters are significantly different $(p<0.05)$. $n$ per group is indicated in parentheses.

part of this region corresponds to a fairly circumscribed area containing neurons identifiable by calbindin, gastrin releasing peptide, and substance P content (Morin et al., 1992; Kalsbeek et al., 1993; Bryant et al., 2000; Muscat et al., 2003; Kriegsfeld et al., 2004). The dorsal part of the region corresponds to an area known to express phosphorylated extracellular signal-regulated kinase (Lee et al., 2003; Antle et al., 2005; Yan et al., 2005). The present analysis also demonstrates significant increases in lightinduced FOS in part of the SCN that appears relatively devoid of this protein. Here, FOS is light induced, but the increased expres- 

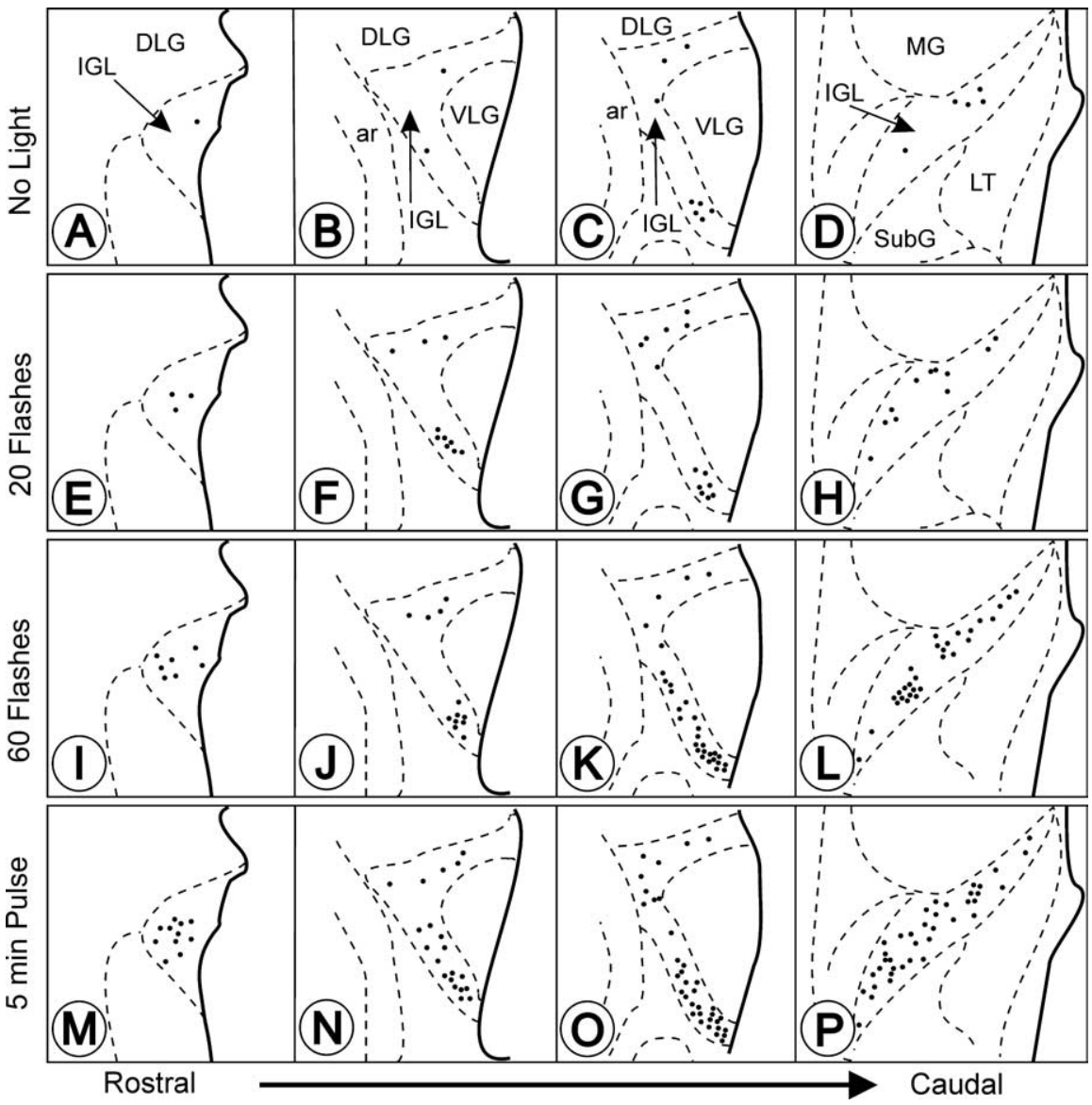

Figure 10. Representative semi-schematic distribution of FOS-IR nuclei in the IGL after various flash presentations over a 5 min interval at circadian time 19. Stimulus conditions are indicated on the left. Each filled circle represents one FOS-IR nuclei. The figure depicts the approximate location of the mean number FOS-IR nuclei counted in the plane of section presented for the indicated stimulus condition. Illustrated sections are separated by $\sim 210 \mu \mathrm{m}$. ar, Acoustic radiation; DLG, dorsal lateral geniculate nucleus; $\mathrm{LT}$, lateral teminal nucleus; MG, medial geniculate nucleus; SubG, subgeniculate nucleus; VLG, ventral lateral geniculate nucleus.

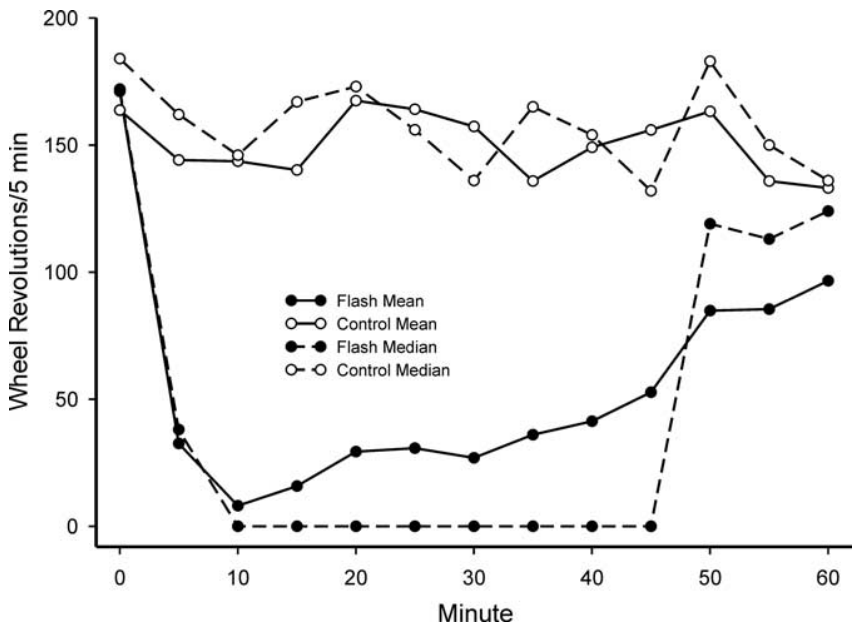

Figure 11. Negative masking of wheel running in response to the standard stimulus (10 flashes $/ 5 \mathrm{~min}$ ). Mean or median revolutions per $5 \mathrm{~min}$ bin are plotted relative to the poststimulus onset time. The revolutions at minute 0 represent the number during the interval before the initiation of the flashes.

sion is less than elsewhere in SCN. A second region of fairly dense light-induced FOS immunoreactivity is present in the dorsal part of the nucleus. This area corresponds to the dorsal extension of the RHT terminal plexus from the SCN into the subparaventricu- lar region and more caudal hypothalamus (Muscat et al., 2003). The light flash or pulse-induced FOS in the subparaventricular region is consistent with results from rat and grass rat (Nunez et al., 1999).

FOS immunoreactivity in IGL neurons appears to require a greater number of flashes than in SCN neurons $(20-40$ vs $10-20 / 5 \mathrm{~min}$ ). This contrasts with previous data showing that light pulse induction of FOS immunoreactivity in IGL cells occurs at much lower irradiances than in SCN neurons (Muscat and Morin, 2006). The anatomical distributions of FOS cells in the IGL after the flash or light pulse stimuli were similar to a previous report (Muscat and Morin, 2006). Despite the dense, fairly uniform distribution of retinal terminals in the leaflet portion of the IGL (Muscat et al., 2003), most of the flash-induced FOS immunoreactivity was evident in the caudal and ventromedial portion of the nucleus.

\section{Masking}

Suppression of wheel running by light (masking) is under the influence of both classical photoreceptors and pRGCs (Mrosovsky et al., 2001; Hattar et al., 2003; Mrosovsky, 2003; Mrosovsky and Hattar, 2003) and correlates well with the effects of light pulses on circadian rhythm phase response. The present studies demonstrate that masking by the standard flash stimulus causes both an acute decline in wheel running and a suppression of running that extends well beyond the actual presence of light. This enduring activitysuppressing effect of light, obscured by typical $1 \mathrm{~h}$ light pulses used for tests of masking, may be an index of continued retinal ganglion cell activity related to the ability of the circadian system to produce large effects on rhythm phase in response to short light pulses (Nelson and Takahashi, 1991; Dkhissi-Benyahya et al., 2000; Muscat and Morin, 2005).

\section{Summary}

The data show that the hamster circadian visual system is highly responsive to brief flash stimuli and that the responses are not predictable according to previously suggested mechanisms inferred from the effects of longer light pulses. Energy integration over time is not as expected. The use of flash stimuli may facilitate understanding of the relative contribution of classical photoreceptors and pRGCs to circadian rhythm regulation.

\section{References}

Amir S, Robinson B (1995) Ultraviolet light entrains rodent suprachiasmatic nucleus pacemaker. Neuroscience 69:1005-1011.

Antle MC, Kriegsfeld LJ, Silver R (2005) Signaling within the master clock of the brain: localized activation of mitogen-activated protein kinase by gastrin-releasing peptide. J Neurosci 25:2447-2454.

Arvanitogiannis A, Amir S (1999) Resetting the rat circadian clock by ultrashort light flashes. Neurosci Lett 261:159-162.

Baylor DA (1987) Photoreceptor signals and vision. Proctor lecture. Invest Ophthalmol Vis Sci 28:34-49. 
Berson DM, Dunn FA, Takao M (2002) Phototransduction by retinal ganglion cells that set the circadian clock. Science 295:1070-1073.

Bryant DN, LeSauter J, Silver R, Romero MT (2000) Retinal innervation of calbindin-D28K cells in the hamster suprachiasmatic nucleus: ultrastructural characterization. J Biol Rhythms 15:103-111.

Dacey DM, Liao HW, Peterson BB, Robinson FR, Smith VC, Pokorny J, Yau KW, Gamlin PD (2005) Melanopsin-expressing ganglion cells in primate retina signal colour and irradiance and project to the LGN. Nature 433:749-754.

Dkhissi-Benyahya O, Sicard B, Cooper HM (2000) Effects of irradiance and stimulus duration on early gene expression (Fos) in the suprachiasmatic nucleus: temporal summation and reciprocity. J Neurosci 20:7790-7797.

Ecker JL, Mackes JL, Lall G, Kuruvilla R, Lucas RJ, Hattar S (2005) Individual contributions of rods, cones, and melanopsin-containing retinal ganglion cells to non-image-forming visual functions. Soc Neurosci Abstr 35:767.3.

Fu Y, Zhong H, Wang M-HH, Luo Dg, Liao H-W, Maeda H, Hattar S, Frishman LJ, Yau K-W (2005) Intrinsically photosensitive retinal ganglion cells detect light with a vitamin A-based photopigment, melanopsin. Proc Natl Acad Sci USA 102:10339-10344.

Fulton AB, Hansen RM (2003) Recovery of the rod photoresponse in infant rats. Vision Res 43:3081-3085.

Hamada T, LeSauter J, Venuti JM, Silver R (2001) Expression of Period genes: rhythmic and non-rhythmic compartments of the suprachiasmatic nucleus pacemaker. J Neurosci 21:7742-7750.

Hattar S, Liao HW, Takao M, Berson DM, Yau KW (2002) Melanopsincontaining retinal ganglion cells: architecture, projections, and intrinsic photosensitivity. Science 295:1065-1070.

Hattar S, Lucas RJ, Mrosovsky N, Thompson S, Douglas RH, Hankins MW, Lem J, Biel M, Hofmann F, Foster RG, Yau KW (2003) Melanopsin and rod-cone photoreceptive systems account for all major accessory visual functions in mice. Nature 424:76-81.

Hendrickson AE, Wagoner N, Cowan WM (1972) An autoradiographic and electron microscopic study of retinohypothalamic connections. Zeitschrift fur Zellforschung 135:1-26.

Hetling JR, Pepperberg DR (1999) Sensitivity and kinetics of mouse rod flash responses determined in vivo from paired-flash electroretinograms. J Physiol (Lond) 516:593-609.

Jacobs GH, Neitz J, Deegan II JF (1991) Retinal receptors in rodents maximally sensitive to ultraviolet light. Nature 353:655-656.

Johnson RF, Morin LP, Moore RY (1988a) Retinohypothalamic projections in the hamster and rat demonstrated using cholera toxin. Brain Res 462:301-312.

Johnson RF, Moore RY, Morin LP (1988b) Loss of entrainment and anatomical plasticity after lesions of the hamster retinohypothalamic tract. Brain Res 460:297-313.

Kalsbeek A, Teclemariam-Mesbah R, Pevet P (1993) Efferent projections of the suprachiasmatic nucleus in the golden hamster (Mesocricetus auratus). J Comp Neurol 332:293-314.

Kriegsfeld LJ, Leak RK, Yackulic CB, LeSauter J, Silver R (2004) Organization of suprachiasmatic nucleus projections in Syrian hamsters (Mesocricetus auratus): an anterograde and retrograde analysis. J Comp Neurol 468:361-379.

Lee HS, Nelms JL, Nguyen M, Silver R, Lehman MN (2003) The eye is necessary for a circadian rhythm in the suprachiasmatic nucleus. Nat Neurosci 6:111-112.

Meijer JH, Groos GA, Rusak B (1986) Luminance coding in a circadian pacemaker: the suprachiasmatic nucleus of the rat and the hamster. Brain Res 382:109-118.
Moore RY, Eichler VB (1972) Loss of a circadian adrenal corticosterone rhythm following suprachiasmatic lesions in the rat. Brain Res 42:201-206.

Moore RY, Lenn NJ (1972) A retinohypothalamic projection in the rat. J Comp Neurol 146:1-14.

Morin LP, Blanchard JH, Moore RY (1992) Intergeniculate leaflet and suprachiasmatic nucleus organization and connections in the hamster. Vis Neurosci 8:219-230.

Mrosovsky N (2003) Contribution of classic photoreceptors to entrainment. J Comp Physiol A Neuroethol Sens Neural Behav Physiol 189:69-73.

Mrosovsky, N, Hattar S (2003) Impaired masking responses to light in melanopsin knockout mice. Chronobiol Int 20:988-999.

Mrosovsky N, Lucas RJ, Foster RG (2001) Persistence of masking responses to light in mice lacking rods and cones. J Biol Rhythms 16:585-588.

Muscat L, Morin LP (2005) Binocular contributions to the responsiveness and integrative capacity of the circadian rhythm system to light. J Biol Rhythms 20:513-525.

Muscat L, Morin LP (2006) Intergeniculate leaflet: contributions to photic and non-photic responsiveness of the hamster circadian system. Neuroscience 140:305-320.

Muscat L, Huberman AD, Jordan CL, Morin LP (2003) Crossed and uncrossed retinal projections to the hamster circadian system. J Comp Neurol 466:513-524.

Nelson DE, Takahashi JS (1991) Sensitivity and integration in a visual pathway for circadian entrainment in the hamster (Mesocricetus auratus). J Physiol (Lond) 439:115-145.

Nunez AA, Bult A, McElhinny TL, Smale L (1999) Daily rhythms of Fos expression in hypothalamic targets of the suprachiasmatic nucleus in diurnal and nocturnal rodents. J Biol Rhythms 14:300-306.

Panda S, Sato TK, Castrucci AM, Rollag MD, DeGrip WJ, Hogenesch JB, Provencio I, Kay SA (2002) Melanopsin (Opn4) requirement for normal light-induced circadian phase shifting. Science 298:2213-2216.

Provencio I, Foster RG (1995) Circadian rhythms in mice can be regulated by photoreceptors with cone-like characteristics. Brain Res 694:183-190.

Provencio I, Rodriguez IR, Jiang G, Hayes WP, Moreira EF, Rollag MD (2000) A novel human opsin in the inner retina. J Neurosci 20:600-605.

Ruby NF, Brennan TJ, Xie X, Cao V, Franken P, Heller HC, O’Hara BF (2002) Role of melanopsin in circadian responses to light. Science 298:2211-2213.

Rusak B (1977) The role of the suprachiasmatic nuclei in the generation of circadian rhythms in the golden hamster, Mesocricetus auratus. J Comp Physiol 118:145-164.

Stephan FK, Zucker I (1972) Circadian rhythms in drinking behavior and locomotor activity of rats are eliminated by hypothalamic lesions. Proc Natl Acad Sci USA 69:1583-1586.

Takahashi JS, DeCoursey PJ, Bauman L, Menaker M (1984) Spectral sensitivity of a novel photoreceptive system mediating entrainment of mammalian circadian rhythms. Nature 308:186-188.

van den Pol AN, Cao V, Heller HG (1998) Circadian system of mice integrates brief light stimuli. Am J Physiol Regul Integr Comp Physiol 275:R654-R657.

Wong KY, Dunn FA, Berson DM (2005) Photoreceptor adaptation in intrinsically photosensitive retinal ganglion cells. Neuron 48:1001-1010.

Yan L, Foley NC, Bobula JM, Kriegsfeld LJ, Silver R (2005) Two antiphase oscillations occur in each suprachiasmatic nucleus of behaviorally split hamsters. J Neurosci 25:9017-9026. 